\title{
Large deletion in PIGL: a common mutational mechanism in CHIME syndrome?
}

José RM Ceroni ${ }^{1}$, Guilherme L Yamamoto ${ }^{1,2}$, Rachel S Honjo ${ }^{1}$, Chong A Kim ${ }^{1}$, Maria R Passos-Bueno ${ }^{2}$ and Débora R Bertola ${ }^{1,2}$

${ }^{1}$ Genetics Unit, Instituto da Criança do Hospital das Clínicas, Faculdade de Medicina, Universidade de São Paulo, São Paulo, SP, Brazil.

${ }^{2}$ Centro de Pesquisa sobre o Genoma Humano e Células-Tronco, Instituto de Biociências, Universidade de São Paulo, São Paulo, SP, Brazil.

\begin{abstract}
CHIME syndrome is an extremely rare autosomal recessive multisystemic disorder caused by mutations in PIGL. PIGL is an endoplasmic reticulum localized enzyme that catalyzes the second step of glycosylphosphatidylinositol (GPI) biosynthesis, which plays a role in the anchorage of cell-surface proteins including receptors, enzymes, and adhesion molecules. Germline mutations in other members of GPI and Post GPI Attachment to Proteins (PGAP) family genes have been described and constitute a group of diseases within the congenital disorders of glycosylation. Patients in this group often present alkaline phosphatase serum levels abnormalities and neurological symptoms. We report a CHIME syndrome patient who harbors a missense mutation c.500T > C (p.Leu167Pro) and a large deletion involving the 5' untranslated region and part of exon 1 of PIGL. In CHIME syndrome, a recurrent missense mutation c.500T > C (p.Leu167Pro) is found in the majority of patients, associated with a null mutation in the other allele, including an overrepresentation of large deletions. The latter are not detected by the standard analysis in sequencing techniques, including next-generation sequencing. Thus, in individuals with a clinical diagnosis of CHIME syndrome in which only one mutation is found, an active search for a large deletion should be sought.
\end{abstract}

Keywords: PIGL, CHIME syndrome, GPI biosynthesis, large deletion.

Received: July 23, 2017; Accepted: October 17, 2017.

CHIME syndrome (OMIM \#280000) is an extremely rare autosomal recessive multisystemic disorder, originally described in 1983 [Zunich and Kaye, 1983], who proposed the discovery of a new neuroectodermal syndrome. The acronym CHIME (colobomas, congenital heart defect, migratory ichthyosiform dermatosis, mental retardation, and ear anomalies) was proposed (Shashi et al., 1995) based on the presence of the most consistent clinical manifestations. CHIME syndrome is caused by biallelic mutations in PIGL (Ng et al., 2012). PIGL is an endoplasmic reticulum localized enzyme that catalyzes the second step of glycosylphosphatidylinositol (GPI) biosynthesis, which plays a role in the anchorage of more than 150 cell-surface proteins including receptors, enzymes and adhesion molecules. Germline mutations in other members of PIG family genes, as well as members of Post GPI Attachment to Proteins (PGAP), involved in structural remodeling of GPI after its attachment to proteins, have been described on a recently

Send correspondence to: José Ricardo M. Ceroni Unidade de Genética do Instituto da Criança do Hospital das Clínicas da FMUSP. Av. Dr. Enéas Carvalho de Aguiar, 647, Cerqueira César, São Paulo, SP, Brazil CEP: 05403-000, E-mail: josericardohc@ gmail.com. emerging group of diseases within congenital disorders of glycosylation. The phenotypic spectrum of these disorders is still not completely known, but clinical overlap between the majority of the disorders in this group is recognized, as patients often present alkaline phosphatase (ALP - which is a GPI-anchored protein) serum levels abnormalities and neurological symptoms, including seizures, hypotonia and mental retardation (Belet et al., 2014). To date, eight probands with mutations in PIGL have been reported, six of them with the diagnosis of CHIME syndrome ( $\mathrm{Ng}$ et al., 2012, Knight Johnson et al., 2017), and two of them presenting clinical findings compatible with Mabry syndrome, characterized by mental retardation and hyperphosphatasia (Fujiwara et al., 2015, Pagnamenta et al., 2017). A recurrent missense mutation c.500T $>C$ (p.Leu167Pro) has been found in heterozygosity in all patients with CHIME syndrome, associated with a null mutation in the other allele, including here two large deletions of different sizes. In contrast, in the two patients with the diagnosis of Mabry, two null mutations were retrieved in PIGL.

We report on an individual presenting a full-blown phenotype of CHIME syndrome that harbors, besides the 
common missense mutation in one allele, a large deletion involving the 5' untranslated region and part of exon 1 of PIGL in the opposite allele. This report gives further support to the fact that large deletions could be a common type of mutations in the second allele in individuals with CHIME syndrome.

The proband is a 4 year-old boy, the first and only child of non-consanguineous parents. During the pregnancy, fetal ultrasound disclosed unilateral hydronephrosis. He was born preterm $(35 \mathrm{w})$, with a birth weight of $3150 \mathrm{~g}$, length of $48 \mathrm{~cm}$ and Apgar scores of 9/10. He evolved with respiratory distress and sepsis, requiring hospitalization for 21 days. His developmental milestones were delayed: he sat unsupported by 10 months of age, walked at 22 months of age, and he has no speech at the age of 4 . He has epilepsy, with a first seizure at 8 months of age during a febrile episode. A partial control of the seizures was obtained with the use of two anti-epileptic drugs. The parents stated that his skin has always been dry and he developed hyperpigmented/ichthyosiform lesions at the age of 1 year. Physical examination at 1 year 9/12 showed: weight of $16 \mathrm{Kg}(\mathrm{p}>$ $95^{\text {th }}$ centile), Height of $94 \mathrm{~cm}\left(\mathrm{p}>95^{\text {th }}\right.$ centile), OFC of $52 \mathrm{~cm}\left(\mathrm{p}>98^{\text {th }}\right.$ centile); fine hair, which is sparse in the temporal regions, prominent forehead, ocular hypertelorism, upslanting palpebral fissures, everted lower lip, small, widely-spaced teeth, with fusion of the central and lateral right incisors, and uplifted ear lobes; short neck; large hands, with brachydactyly, finger pads and incomplete single palmar creases; hyperchromic/ichthyosiform skin lesions in neck, axillas and knees, geographic lesions with hyperpigmented borders in the thorax and abdominal areas and palmar hyperkeratosis (Figure 1). Complementary exams disclosed: normal echocardiography and cranial CT scan; hand X-rays with short distal phalanges of all fingers, as well, as the medial phalanx of the left fifth digit, and advanced bone age; ophthalmologic evaluation, bilateral retinal coloboma; renal ultrasound, bilateral pyelocaliceal ectasia; alkaline phosphatase (ALP) serum levels of $456 \mathrm{U} / \mathrm{L}$ and $319 \mathrm{U} / \mathrm{L}$ (normal range 150-380 U/L); pyridoxal-phosphate $27.2 \mathrm{ug} / \mathrm{L}$ (normal range $5.2-34 \mathrm{ug} / \mathrm{L}$ ); normal blood and skin G-banded karyotype and chromosomal microarray. No formal audiologic evaluation was performed. As the patient showed clinical features compatible with the diagnosis of CHIME syndrome, whole exome sequencing (WES) was performed and the recurrent heterozygous mutation p.Leu167Pro in PIGL was identified. In order to seek for the second mutation, we analyzed this gene with the Integrative Genomics Viewer (IGV) and a deletion of 802 base pairs ([hg19] chr7: 16,119,889$16,120,690$ ) involving the 5 ' untranslated region and the first 50 aminoacids, including the ATG start codon in exon 1 was observed (Figure 2). This gene alteration was confirmed by bridging PCR and Sanger sequencing (Fig. 1). Clinical and molecular data of the present patient, as well as the other individuals reported in literature with mutations in
PIGL are shown in Table 1 (Zunich and Kaye, 1983, Shashi et al., 1995, Tinschert et al., 1996, Schnur et al., 1997, Sidbury and Paller, 2008, Ng. et al., 2012, Fujiwara et al., 2015, Knight Johnson et al., 2017, Pagnamenta et al., 2017).

PIGL mutations have been reported mainly in individuals with CHIME syndrome (eight individuals, including a pair of brothers and the present case), one individual reported by Fujiwara (Fujiwara et al., 2015), clinically diagnosed as Mabry syndrome, and one individual recently reported by Pagnamenta (Pagnamenta et al., 2017) whose clinical and laboratorial findings were also reminiscent of Mabry syndrome. In the eight individuals with CHIME syndrome with PIGL proven mutations (Ng et al., 2012, Fujiwara et al., 2015, Knight Johnson et al., 2017, Pagnamenta et al., 2017), we could retrieve a homogeneous phenotype, characterized by developmental delay/intellectual disability, epilepsy, ocular coloboma, conductive hearing loss and icthyosiform skin rash in all of them. In the neonatal period, some malformations could be observed by fetal ultrasssonography in a few patients (3/9), such as cardiac abnormality, cleft lip/palate, and hydronephrosis. The majority of the individuals were born at term, with high birth weight and length. The most frequent complications found in the neonatal period were respiratory distress $(2 / 9)$ and hypoglicemia (2/9). Nevertheless, overgrowth was not frequently observed in the evolution: only two individuals presented with weight and/or height greater than 2SD above the mean. Facial dysmorphisms were represented

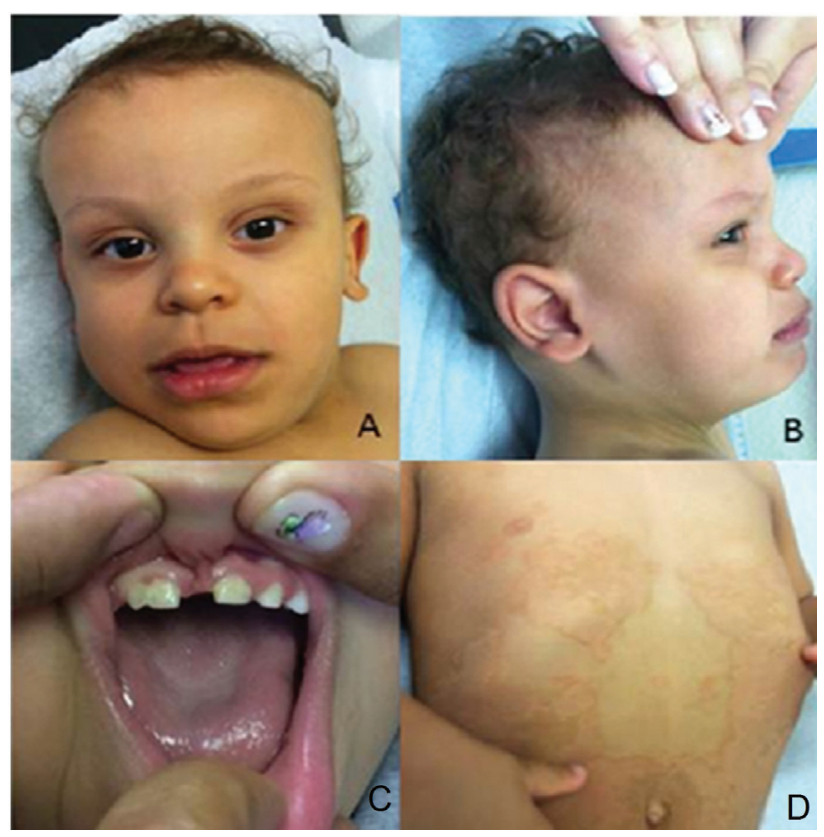

Figure 1 - A-D. Fine, sparse hair in the temporal regions, proeminent forehead, ocular hypertelorism, upslanting palpebral fissures, everted lower lip, small, widely-spaced teeth, diastema and fusion of the central and lateral right inceisors, uplifted ear lobes; short neck; hyperchromic/icthyosiform skin lesions, geographic lesions with hyperpigmented borders in the thorax and abdominal areas. 


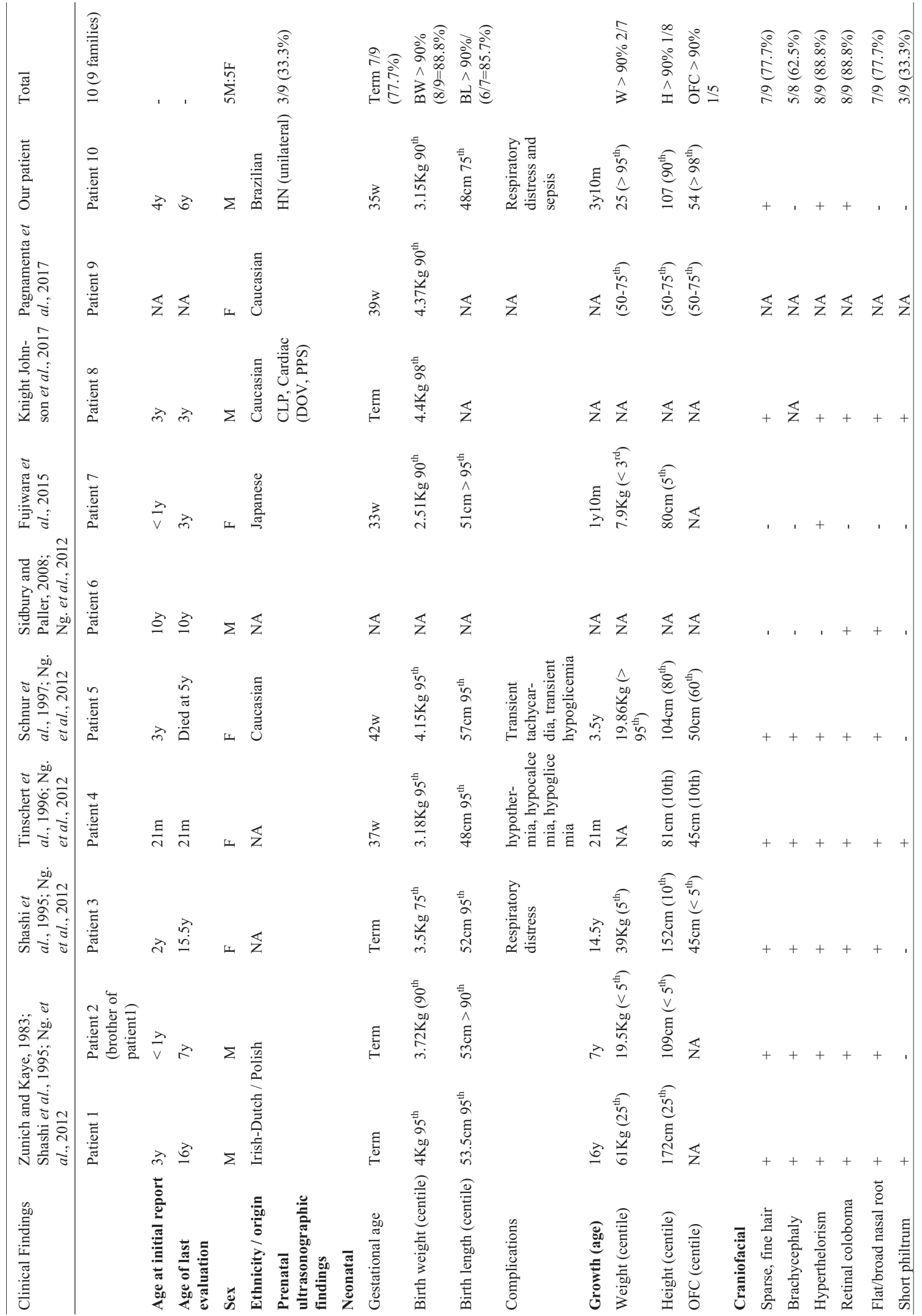




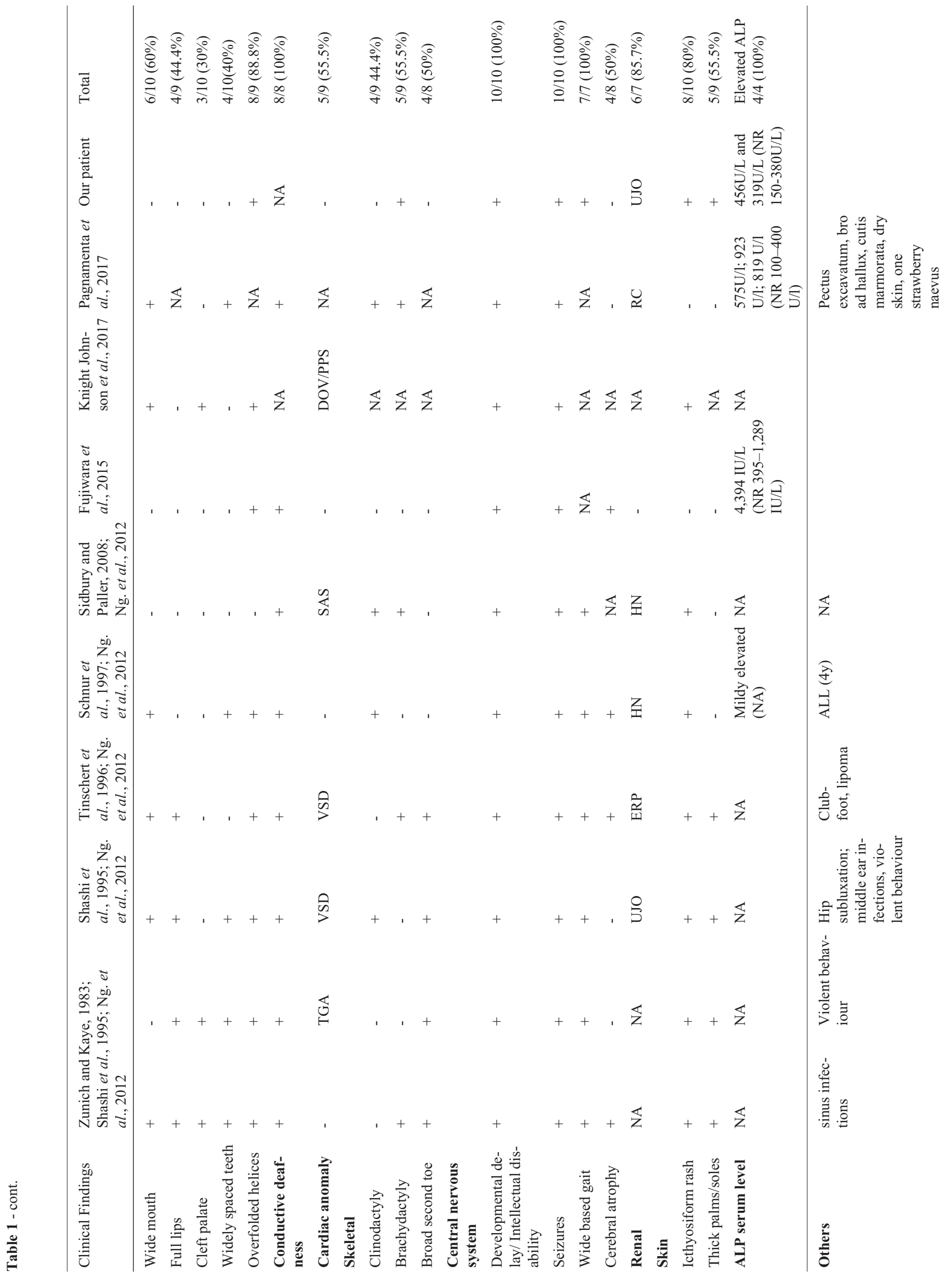


mainly by sparse, fine hair, ocular hypertelorism, flat/broad nasal root, ears with overfolded helices. Different structural cardiac abnormalities were observed in 5/9, including isolated VSD, valvar stenosis and outflow tract defects, as well as renal anomalies (6/7), mainly hydronephrosis. Clinodactyly/brachydactyly and thickness of the palms and soles could be observed in approximately $50 \%$ of the cases. One individual developed acute lymphoblastic leukemia (ALL) at the age of 4 . This is the only malignant neoplastic condition described in CHIME individuals. As this hematological abnormality is common in the pediatric population, we cannot exclude that this association could be fortuitous. ALP serum levels have not been routinely measured in patients harboring PIGL mutations, with only one patient reported as presenting mild elevation (Schnur et al., 1997). The present patient also showed mild elevations on two different occasions. The degree of abnormality in the ALP serum levels is partly explained by the position of the enzyme in the GPI biosynthesis pathway: proteins responsible for the latter steps are prone to lead to higher ALP serum levels, as this is seen in Mabry syndrome, mainly caused by mutations in PIGV (Krawitz et al., 2010, Murakami et al., 2012). In contrast, PIGL, responsible for the second step, lead to mild elevations (Murakami et al., 2012). However, high ALP serum levels were retrieved in two individuals harboring biallelic null mutations (frameshift and/or nonsense) in PIGL (Fujiwara et al., 2015, Pagnamenta et al., 2017), indicating that, not only the physical localization of these different enzymes in the GPI biosynthesis pathway are important to determine the secretion of GPI-anchored proteins, but also their residual functional activity.

In all the eight probands described so far with CHIME syndrome, in which the molecular analysis was performed, the missense mutation c.500T $>\mathrm{C}$ (p.Leu167Pro) was present in one allele, combined with a null mutation in the opposite allele: frameshift mutation (1), nonsense mutation (1), splicing mutation (1), and large deletions (3). In one individual, the authors failed to identify the second mutation. Although the number of patients with proven mutations in PIGL is too small to draw definitive conclusions, we could observe an overrepresentation of large deletions, which sizes ranged from $802 \mathrm{bp}$, as the one found in the present patient, to $1 \mathrm{Mb}$ deletion in $17 \mathrm{p} 12-$ 11.2, encompassing the whole gene (Tinschert et al., 1996). In the patient reported by Knight Johnson (Knight Johson et al., 2017), the breakpoints of the deletion involving PIGL exons 4 to 6 occurred within Alu-repeats. As these Alu sequences were overrepresented in the intronic regions of $P I G L$, the authors suggested that copy number variations could occur as a consequence of this specific genomic architecture. The breakpoints of the deletion presented by our patient do not follow this rule, as one of them occurs within exon 1. Large deletions are not detected by the standard analysis of sequencing techniques, including next-generation sequencing. This could be the case in the patient re- 


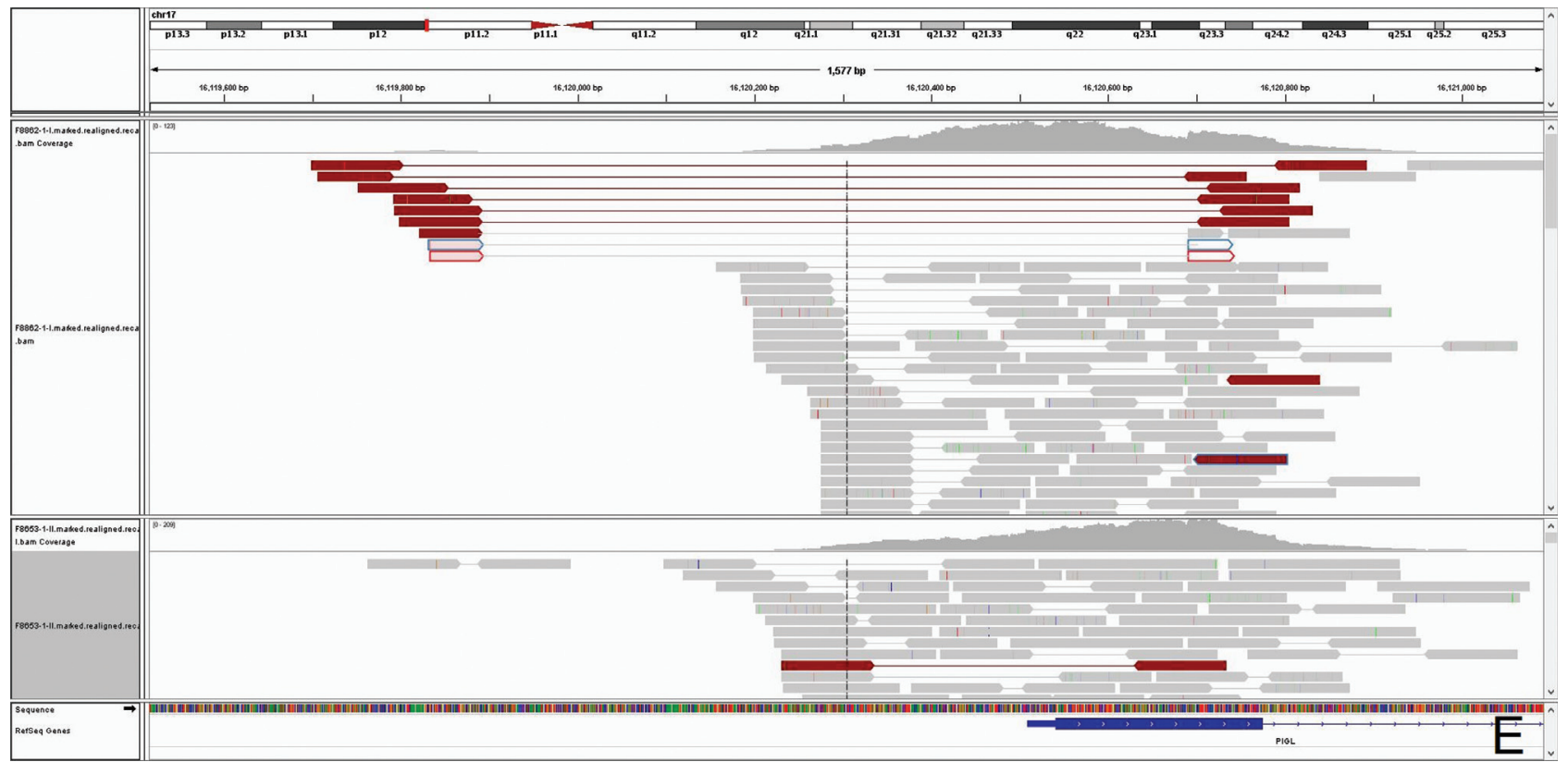

Figure 2 - IGV analysis of PIGL showing a deletion of 802 base pairs ([hg19] chr7: 16,119,889-16,120,690) involving the 5' untranslated region and the first 50 aminoacids, including the ATG start codon in exon 1.

ported by $\mathrm{Ng}(\mathrm{Ng}$ et al., 2012) in which only one mutation was identified. Thus, in individuals with a clinical diagnosis of CHIME syndrome in which only one mutation is found, especially the recurrent p.Leu167Pro, an active search for a large deletion should be sought.

The whole phenotypic and genotypic spectrum in individuals harboring mutations in PIGL is still incompletely characterized. Interestingly, individuals presenting two null mutations in PIGL did not present the typical CHIME syndrome phenotype. Instead, their phenotype was compatible with the diagnosis of Mabry syndrome, with severe neurological involvement and high serum levels of APL. Thus, the residual protein function coded by PIGL is important in the phenotypic delineation. It remains to be determinated if the presence of two less severe mutations, such as, two missense mutations, would lead to a mild phenotype or even lack of phenotypic expression. Further reports are required to clarify this matter.

\section{Acknowledgments:}

We would like to thank the family for their participation in this study. This study was supported by FAPESP (CEPID 2013/08028-1); and CNPq (302605/2013-4).

\section{References}

Belet S, Fieremans N, Yuan X, Van Esch H, Verbeeck J, Ye Z, Cheng L, Brodsky BR, Hu H, Kalscheuer VM, et al. (2014) Early frameshift mutation in PIGA identified in a large XLID family without neonatal lethality. Hum Mutat 3:350-355.

Fujiwara I, Murakami Y, Niihori T, Kanno J, Hakoda A, Sakamoto O, Okamoto N, Funayama R, Nagashima T, Nakayama K, et al. (2015) Mutations in PIGL in a patient with Mabry syndrome. Am J Med Genet A 167:777-785.

Knight Johnson A, Schaefer GB, Lee J, Hu Y and Del Gaudio D (2017) Alu-mediated deletion of PIGL in a Patient with CHIME syndrome. Am J Med Genet A 173:1378-1382.

Krawitz PM, Schweiger MR, Rödelsperger C, Marcelis C, Kölsch U, Meisel C, Stephani F, Kinoshita T, Murakami Y, Bauer S, et al. (2010) Identity-by-descent filtering of exome sequence data identifies PIGV mutations in hyperphosphatasia mental retardation syndrome. Nat Genet 42:827-829.

Murakami Y, Kanzawa N, Saito K, Krawitz PM, Mundlos S, Robinson PN, Karadimitris A, Maeda Y and Kinoshita T (2012) Mechanism for release of alkaline phosphatase caused by glycosylphosphatidylinositol deficiency in patients with hyperphosphatasia mental retardation syndrome. J Biol Chem 287:6318-6325.

Ng BG, Hackmann K, Jones MA, Eroshkin AM, He P, Wiliams R, Bhide S, Cantagrel V, Gleeson JG, Paller AS, et al. (2012) Mutations in the glycosylphosphatidylinositol gene PIGL cause CHIME syndrome. Am J Hum Genet 90:685-688.

Pagnamenta AT, Murakami Y, Taylor JM, Anzilotti C, Howard MF, Miller V, Johnson DS, Tadros S, Mansour S, Temple IK, et al. (2017) Analysis of exome data for 4293 trios suggests GPI-anchor biogenesis defects are a rare cause of developmental disorders. Eur J Hum Genet.25:669-679.

Schnur RE, Greenbaum BH, Heymann WR, Christensen K, Buck AS and Reid CS (1997) Acute lymphoblastic leukemia in a child with the CHIME neuroectodermal dysplasia syndrome. Am J Med. Genet 72:24-29.

Shashi V, Zunich J, Kelly TE and Fryburg JS (1995) Neuroectodermal (CHIME) syndrome: an additional case with long term follow up of all reported cases. J Med Genet 32:465-469.

Sidbury R and Paller AS (2008) What syndrome is this? Pediat Dermatol 18:252-254. 
Tinschert S, Anton-Lamprecht I, Albrecht-Nebe $\mathrm{H}$ and Audring $\mathrm{H}$ (1996) Neuroectodermal syndrome: migratory ichthyosiform dermatosis, colobomas, and other abnormalities. Pediat Derm 13:363-371.
Zunich J and Kaye CI (1983) New syndrome of congenital ichthyosis with neurologic abnormalities. Am J Med Genet $15: 331-333$.

Associate Editor: Mara H. Hutz

License information: This is an open-access article distributed under the terms of the Creative Commons Attribution License (type CC-BY), which permits unrestricted use, distribution and reproduction in any medium, provided the original article is properly cited. 